\title{
Crystallization of zinc lactate in presence of malic acid
}

\section{Xiang Yang Zhang(1), Gilles Févotte(2), Liang Zhong(1), GANG Qian(1), XiNG-Gui ZHOUA $^{(1) *}$, WEI-KANG YUAN(1)}

(1) East China University of Science and Technology, State Key Laboratory of Chemical Engineering, 200237 Shanghai, China

(2) Ecole Nationale Supérieure des Mines de Saint Etienne ; Centre SPIN ; Département GENERIC ; LPMG -UMR CNRS 5148 ; 158 Cours Fauriel, 42023 Saint-Étienne Cedex 2, France

\begin{abstract}
The influence of malic acid, which acts as an impurity on the cooling crystallization of zinc lactate is investigated in this paper by monitoring the relative supersaturation and the number of crystals during crystallization. The presence of malic acid increases the solution solubility and makes the metastable zone wider; it also changes the habit of the crystal. The purity of the final products is shown to be influenced by the amount and size of seed crystals, cooling rate, seeding temperature and final temperature, but appears to depend mainly on the particle size and level of supersaturation. Residual supersaturation thresholds are observed that depend on the final temperature. A model is proposed to predict the steady-state supersaturation value from the final temperature at a given impurity concentration. This model is based on Kubota and Gibbs equations.
\end{abstract}

Keywords:

A1. Impurities; A1. Industrial crystallization; A1. Purification; B1. Zinc lactate; B1. Malic acid

\section{Introduction}

Crystallization is an important separation and purification process used widely in food, fine chemical and pharmaceutical industries [1]. Especially in the pharmaceutical industry, crystallization processes are often carried out as the final purification step in the manufacturing of active pharmaceutical ingredients (APIs), enabling one to obtain solid products with high purity at low processing costs [2].

Crystallization is a very complicated process as it involves many concomitant phenomena such as nucleation, growth, breakage and agglomeration of crystals, and the purity and physical properties (morphology, crystal size distribution, bulk density, product filterability, and dry solid flow properties) of the crystals are significantly influenced by the selection of the crystallization process (e.g. cooling, evaporation, or antisolvent addition) [3] and [4]. The control of manipulated "input" variables during the crystallization process (e.g. stirring, cooling or evaporation rates, seed amount, rate of antisolvent addition, etc.) also plays a keyrole in the determination of the final dispersed solid properties. Despite the long history and widespread application of crystallization, a large number of unsolved problems remain concerning modeling, design and control of the industrial processes, which require an indepth and improved understanding of the mechanisms of nucleation, growth, breakage and agglomeration, and of the influence of the process variables on these mechanisms.

\footnotetext{
*Corresponding author: xgzhou@ecust.edu.cn
} 
Industrial solutions are almost invariably impure, by any definition of the term, and in many cases small amounts of impurities present in the solution were reported to have a dramatic effect on solubility, nucleation, crystal growth and morphology [4]. Furthermore, chemical purity is probably the most important index of product quality, and is obviously of paramount concern for pharmaceuticals because small amounts of impurities are likely to result in reduced drug efficacy and harmful side effects. Understanding the detrimental effect of impurity on the crystallization and controlling the process so as to reduce the impurity content of the main crystallizing product is therefore a very significant engineering issue.

The effect of impurities on the development of crystallization is rather complex and difficult to foresee, due to many mechanisms which are likely to govern the crystal/impurity interactions. The capture of impurities in the crystals during the growth in solution clearly results from combined effects of many factors like the chemical composition of solution, the relative solubility of host and impurity phases, interactions between host and impurity molecules, relative dimensions of substituting (impurity) and substituted (host phase) ions, the similarity in crystallographic structure of the two phases, and the crystallization conditions [5] and [6].

Among other phenomena, trace impurities can have highly unexpected effects on equilibrium solubility measurements. For example, the solubility of potassium sulphate is significantly lowered when measured by dissolving $\mathrm{K}_{2} \mathrm{SO}_{4}$ crystals in water containing parts per million traces of $\mathrm{Cr}$ (III) [7]. As a consequence, it is clear that solubility variations can change the final yield of crystallization operations through changes of the steady-state solute concentration and supersaturation [4].

Impurities can also be captured as liquid inclusions into the crystal or incorporated in the crystal lattice. According to Zhang [8], the nature and location of the guest molecules in the lattice of the host crystals are not well understood. The presence of liquid inclusions generally results in detrimental effects such as caking and agglomeration of the particles on storage, or release of undesirable compounds upon dissolution.

Actually, incorporation of impurities into the crystal lattice through molecular substitution seems to be the most common mechanism of impurity contamination [8] and [9]. Thermodynamic factors may allow the co-crystallization of an impurity, which, especially when the crystallization process is slow, is governed by equilibrium concentration conditions. In this case, the incorporation of impurity can be described by a segregation coefficient, assuming, e.g. regular solution behavior [10].

However, according to Meenan et al. [4], normally, the amount of impurities incorporated in the crystalline phase significantly exceeds the amount predicted by thermodynamics. Such worsening of the incorporation of impurities is usually assumed to be related to nonequilibrium growth conditions and, consequently, to kinetic features of the crystallization process. The latter being obviously exacerbated by the fast crystallization rates required during industrial manufacturing processes. It is clear that under non-equilibrium conditions, the kinetics of crystal formation is likely to have a large impact on the product purity.

Complex effects of impurity species on the crystal growth rates were also observed: reduced growth rates and final residual supersaturation thresholds [11] and [12]. The onset of nucleation can be made easier or delayed by impurities effects, or unaffected by compounds that, on the other hand, are reported to alter crystal growth rates [13] and [14]. As an example, the latter paper demonstrates that among six different cations impurities introduced during the solution crystallization of ammonium oxalate, three of them lead to delayed primary nucleation (i.e. an increase of the so-called metastable zone width: Mn(II), Co(II), $\mathrm{Ni}(\mathrm{II})$ ), while the other three impurities (Cu(II), Fe(III), Cr(III)) do not appear to have any effect on the nucleation rate. It therefore seems that there is an intimate relationship between the effect of impurity on nucleation and growth kinetics and on their incorporation in the crystal lattice. Both would be results of interactions between solute/impurity species and crystallizing particles [12]. However, it is worth noting that such complex and coupled effects remain rather poorly explored and that most experimental observations cannot clearly be described by the currently available kinetic models. In order to illustrate this latter remark, it is interesting to note that through the Population Balance Equation (PBE) dynamic 
simulation of crystallization processes, one can clearly show that any change in the growth rate impacts on the experimental observation of the onset of primary nucleation (i.e. the end of the so-called "induction time"). Indeed, the population density of newly generated particles is given by the boundary condition $\psi\left(L_{\min , t} t\right)=R_{\mathrm{N}}(t) / G(t)$, where $L_{\min }$ is the critical size of nuclei, $R_{N}$ and $G$ are the nucleation and growth rates of crystals, respectively. Such coupled feature of nucleation and growth phenomena makes the joint observation of constant nucleation rate together with reduced growth rate rather unclear and questionable, and raises a question about the consistency of available kinetic models describing crystallization phenomena in the presence of impurities.

Commonly, two steps are assumed to take place during the incorporation of impurities into the crystals and it is very likely that such incorporation results in the creation of local defects in regular crystal lattice, especially if the impurity species differs in size. The impurities first adsorb on the growing crystals surfaces, and then incorporate into the crystal lattice [4]. The first step was often explained by the surface adsorption theory. It was proposed that impurities could be adsorbed selectively on specific faces, thus slowing down the growth of the same faces by hindering the attachment of additional molecules [15], [16] and [17]. Such a mechanism clearly results in a change of the crystal shape. Especially, the tailor-made impurity, which can be adsorbed only on a symmetry subset of crystallographic surface sites on specific faces via host-like parts of their molecule, was often applied for the crystal habit modification [18].

Up to now a limited number of papers address the influence of process conditions on the quality of the final products, especially on the purity of the crystals, which do not match with the significance of the problem [19]. In addition, to the best of our knowledge, the dependence of temperature on the above mentioned final residual supersaturation threshold was never assessed in previous experimental studies.

This paper presents preliminary experimental results obtained during the study of the crystallization of zinc lactate in the presence of racemic malic acid, which was selected as a model impurity. The variations of solubility and limit of metastable zone of zinc lactate in the presence of malic acid was first evaluated. Then cooling crystallization operations of zinc lactate in impure media were carried out under varying operating conditions in order to investigate the relationship between secondary nucleation and crystal growth rate on the one hand, and purity, particle size, yield and crystal structure of the final products, on the other hand. The aim of this research is to evaluate possible effects that should be taken into account for industrial crystallization process development purposes and for better mastering the final chemical purity of crystals grown in impure media. This study is therefore a step towards improved modeling, optimization and control of crystallization processes in the presence of impurities.

\section{Experimental}

\section{II.1. Chemicals}

Racemic zinc lactate was obtained as a commercial product and used without purification. The purity was about 98.6\%. Other chemicals including racemic malic acid and EDTA disodium were supplied by Sinopharm Chemical Reagent Co. and Shanghai Lingfeng Chemical Reagent Co., respectively, at an analytical grade (99.0 wt\%). Double-distilled water was used in all the experiments.

In preparatory experiments, it was found that malic acid was easy to incorporate into the final zinc lactate crystals, while propanoic acid, fumaric acid, butanedioic acid and tartaric acid could easily be removed by crystallization. Even though malate ions are bigger than lactate, their incorporation in the crystal lattice is probably made possible by the structural similarity of the two molecules. For this reason, malic acid was selected as the impurity in this work.

\section{II.2. Experimental apparatus}

Laser backscattering, also known as focused beam reflectance measurement (FBRM) (LASENTEC, Mettler Toledo, Swiss), is widely used in the pharmaceutical industry to monitor 
changes in the crystal size distribution (CSD) and number of crystals. However, it is worth noting that the FBRM probe does not measure the "true CSD", but the chord length distribution (CLD) of particles in the slurry [20]. An FBRM therefore allows estimating qualitative changes in the CSD resulting from crystallization phenomena, such as agglomeration, breakage, nucleation, etc. In this study, an FBRM was used to measure the changes in the crystal amount and to detect the occurrence of nucleation. The lower limit of the chord length measurements was $0.5 \mu \mathrm{m}$.

The weight average size of the crystals harvested at the end of the batch cooling crystallization was determined off-line using a "classical" laser diffraction particle size analyzer (Malvern Mastersizer 2000 U.K.).

The crystal habits were observed using scanning electron microscopy (JSM-636oLV, Jeol, Japan) with a magnification of 2000 $\times$. The crystal seeds were observed under an optical microscope (ECLIPSE E200, Nikon, Japan) with a magnification of 100×. The X-ray diffractograms of the crystals were recorded using an RINT200o equipment (Rigaku Japan), in order to verify the possible effect of malic acid on crystal lattice.

A HPLC equipped with a UV detector (HP 1100 America) was used off-line to determine the concentration of malate ions in the final crystals. The chromatographic column was an Agilent ZORBAX SB-C18 $\left(4.6 \times 150 \mathrm{~mm}^{2}\right)$ and the mobile phase was a buffer solution of sodium biphosphate at $0.05 \mathrm{M}$, the $\mathrm{pH}$ value of which was adjusted by phosphoric acid to 2.5 . The velocity of mobile phase was set to be $0.3 \mathrm{~mol} / \mathrm{min}$, and the analysis was conducted at $20{ }^{\circ} \mathrm{C}$ and $210 \mathrm{~nm}$ UV light.

The batch crystallization process was monitored by an in-situ benchtop conductivity meter (FE30, Mettler Toledo) during crystallization [21]. Off-line complexometric titration analysis [22]was used to calibrate the conductivity meter and the zinc lactate concentration was computed from the conductivity measurements by taking into consideration the influences of temperature and solid concentration.

\section{II.3. Experimental setups and procedures}

\section{II.3.1. Determination of solubility curves and limit curves of metastable zone}

To measure the solubility, zinc lactate (with or without malic acid) was dissolved in doubledistilled water, and the suspension was stirred for $4 \mathrm{~h}$ in a double jacketed vessel (Figure 1). The temperature of the vessel was kept constant by circulating water in the jacket from a thermostatic bath. After the stirring was stopped, the suspension was allowed to settle for $2 \mathrm{~h}$ to allow complete sedimentation of the fine crystals. Three samples of about $10 \mathrm{ml}$ each were carefully withdrawn from the clear solution using a $10 \mathrm{ml}$ pipettes equipped with a filter on top so as to make sure that fine crystals were completely removed. The weighed samples of saturated solutions were complexometrically titrated with an EDTA disodium [22].

A total of 17 solubility experimental runs were carried out at temperatures ranging from 5.1 to $87.7^{\circ} \mathrm{C}$ to determine the solubility of pure zinc lactate, and a total of six solubility points were measured at temperature ranging from 32.2 to $77.8^{\circ} \mathrm{C}$ for an impure zinc lactate. The concentration of malic acid in the impure zinc lactate (referred to an anhydrous zinc lactate) was $5.85 \mathrm{wt} \%$. All the solubility and limit of metastable zone data presented in this paper were the average values of three repeated determinations and the accuracy was within $\pm 1 \%$.

The limit of metastable zone was measured on a Labmax system (Mettler Toledo) with a $500 \mathrm{ml}$-crystallizer, as shown in Figure 2. Solutions prepared by mixing fixed amounts of pure or impure zinc lactate with double-distilled water were charged to the vessel, which was sealed to protect from dust and moisture condensation. The solutions were first heated to a temperature higher than that of solubility to allow complete dissolution of the solid, and were then cooled at a fixed constant rate $\left(10^{\circ} \mathrm{C} / \mathrm{h}\right)$ until nucleation occurred. The nucleation point was easily detected by the FBRM probe, and the temperature of nucleation was then recorded

\section{II.3.2.Cooling batch seeded experiments with and without impurities}

The experimental setup for seeded cooling crystallization was the same as used to determine the limit of metastable zone. The stirring rate was kept constant for all the cooling 
crystallization experiments, which was the same as that used to determine the supersaturation limit. Solutions prepared from $32.32 \mathrm{~g}$ zinc lactate, $1.60 \mathrm{~g}$ malic acid and $400 \mathrm{~g}$ double-distilled water were first heated to $85^{\circ} \mathrm{C}$ (higher than the solubility temperature of about $81.5^{\circ} \mathrm{C}$ ) to allow complete dissolution of the solid. The solutions were then cooled to 70 or $75{ }^{\circ} \mathrm{C}$ so as to introduce seeds in the supersaturated region.

Seed crystals were prepared as follows. Pure zinc lactate crystals were milled and sieved. Particles of sizes between 160 and 200 meshes and those smaller than 400 meshes were used as seed crystals. The weight average sizes of the seed crystals were 9.4 and $19.4 \mu \mathrm{m}$, as measured using the laser diffraction particle size analyzer mentioned previously. The amount of seed crystals used was $1 \%$ or $2 \%$ of the total weight of added zinc lactate. Pictures of the seeds are shown in Figure 3, from which one can see that the seed CSD is rather broad and exhibits many fine crystals probably resulting from breakage during the grinding process. Such particles are obviously too small to be efficiently sieved, in particular because of static electricity. Fine seed particles are likely to behave as "false nuclei" at the beginning of the crystallization process.

During the batch crystallization, the temperature of the solution was lowered linearly at specified rates $\left(5{ }^{\circ} \mathrm{C} / \mathrm{h}, 7.7{ }^{\circ} \mathrm{C} / \mathrm{h}\right.$ or $\left.10^{\circ} \mathrm{C} / \mathrm{h}\right)$ to specified values $\left(30^{\circ} \mathrm{C}, 40^{\circ} \mathrm{C}\right.$ or $\left.50^{\circ} \mathrm{C}\right)$. Afterwards, the suspension was maintained at constant temperature and under stirring for $150 \mathrm{~min}$. The variations of solute concentration were monitored by a conductivity analyzer which was off-line calibrated by complexometric titration analysis performed on samples withdrawn during crystallization. The time variations of crystal sizes and number were monitored by the FBRM. After the final temperature was reached, the suspension was continuously stirred for $24 \mathrm{~h}$ in order to ensure the termination of the crystal growth. Suspension samples were finally withdrawn from the crystallizer for analysis. The final solute concentration was analyzed by titration to determine accurately the yield of the batch process, and the impurity content of the final crystals was measured by the HPLC. The weight average crystal size was measured using laser granulometry, the crystal habit was determined using the SEM, and the crystal structure was evaluated by XRD, respectively.

\section{Results and discussion}

\section{III.1. Solubility and metastable zone width}

Figure 4 shows the solubility of zinc lactate in the presence of $5.85 \%$ malic acid as compared to the solubility of pure zinc lactate. The solubility of zinc lactate is increased in the presence of malic acid and the solubility curve determined at a fixed concentration of malic acid is approximately parallel to that of pure zinc lactate. Such results are similar to previously published experimental data [12] and [14]. The effect of malic acid on the solubility of zinc lactate can be explained by changes of $\mathrm{pH}$ and possible formation of metal-organic complexes in the solution. As a matter of fact, organic acids are frequently used to increase the solubilities of minerals in hydrothermal fluids [23] and [24]. Primary nucleation turns out to be delayed when malic acid is present in solution (i.e. the start of concentration decrease related to the initial generation of solid takes place at a higher level of supersaturation). The corresponding limit of metastable zone is known to depend on the solubility, the temperature and the solution/crystal interfacial tension.

\section{III.2. Final purity of the crystals}

Seeded crystallization experiments were carried out to investigate the effect of operating parameters, such as the amount and weight average size of seed crystals ( $W_{\text {seed }}$ and $D_{\text {seed, }}$, respectively), cooling rate, seeding temperature $\left(T_{s}\right)$ and final temperature $\left(T_{\text {final }}\right)$ and final concentration $\left(C_{\text {final }}\right)$ of the mother liquor, final impurity concentration $\left(C_{i}\right.$, wt\% with respect to anhydrous zinc lactate) and weight average size of the crystals $\left(D_{f, e x p}\right)$, crystal growth rate and yield of the batch crystallization process. The results are listed in Table 1. For all the experiments, the initial impurity concentration was $5.85 \mathrm{wt} \%$ (with respect to an anhydrous zinc lactate), and the yield was calculated as 
Yield $=\frac{C_{\text {initial }}-C_{\text {final }}}{C_{\text {initial }}}$

where $C_{\text {initial }}$ is the initial concentration of zinc lactate $\left(\mathrm{g} / 100 \mathrm{~g} \mathrm{H}_{2} \mathrm{O}\right)$.

The variations of zinc lactate concentration, relative supersaturation and crystal amount during these crystallization processes are displayed in Figure 5, Figure 6 and Figure 7, respectively. The relative supersaturation was calculated as follows:

$$
\sigma(t)=\frac{C(t)-C^{*}\left(t, C_{i}\right)}{C^{*}\left(t, C_{i}\right)}
$$

where $C(t)$ is the measured concentration during the crystallization, and $C^{*}\left(t, C_{i}\right)$ the concentration at saturation in presence of malic acid.

Moreover the final concentration and relative supersaturation, which were measured after the suspensions was left under stirring for $24 \mathrm{~h}$ at the final temperature, are indicated by lines $a$, $b, c$ in Figure 5 and Figure 6.

As shown in Table 1, the crystals obtained after the experiments contained small amounts of malic acid. To determine whether the incorporation of malic acid in the final crystals resulted from the inclusion of mother liquid, the final crystals were grinded to less than $10 \mu \mathrm{m}$ and washed several times with ethanol, which easily dissolves malic acid but not zinc lactate. The amount of malic acid in the crushed solids was hardly changed, thus indicating that malic acid had incorporated into the crystal lattice.

In order to analyse the experimental results, additional estimations were made as follows. The corresponding results are displayed in Table 2. First, an SA, a parameter proportional to the seed area was calculated as follows, where $N_{\text {seed }}, D_{\text {seed }}$ and $W_{\text {seed }}$ are the seed number, size and weight, respectively, $\bar{a}_{\text {seed }}$ and $\bar{v}_{\text {seed }}$ are the area and volume of an average seed particle, respectively, $\phi$ is a shape factor

$$
S A \propto N_{\text {seed }} \bar{a}_{\text {seed }} \propto N_{\text {seed }} \phi_{\text {seed }} D_{\text {seed }}^{2} \propto \phi_{\text {seed }} D_{\text {seed }}^{2} \frac{W_{\text {seed }}}{\bar{v}_{\text {seed }}} \propto \frac{W_{\text {seed }}}{D_{\text {seed }}}
$$

As one can see in Table 2, experiments 2-5 correspond to similar seed areas, while the area of seeds was divided and multiplied by two during experiments 6 and 1 .

The capture of impurities in crystals during their growth from solutions depends on a number of factors like the chemical composition of solution, relative solubilities of host and impurity phases, and interactions between host and impurity molecules. When the crystals grow slowly at very low supersaturation, the segregation of an impurity between the liquid phase and the crystalline solid phase at equilibrium can be predicted by thermodynamics, and has been examined by a number of researchers [10], [25], [26] and [27]. If the solid is considered as a solid solution and the solution is in equilibrium with the liquid solution, one can use a segregation coefficient that relates the impurity concentration in the liquid and solid

$$
K i=\frac{x_{\text {solid }}}{x_{\text {liquid }}}
$$

where $x$ is the mass ratio of impurity to the solute. $K i$ has to be smaller than one to have a purification effect by crystallization. $\mathrm{Ki}$ is constant during crystallization by evaporation or becomes larger at lower temperatures during crystallization. In any case, the impurity will accumulate in the mother liquid when solid is crystallized out of it, and the impurity concentration in the liquid will be higher when more solute goes to solid. For a constant segregation coefficient $K i$ in evaporation crystallization, more impurity will go to solid. This is also true when an impurity concentration in the liquid is increased by cooling crystallization. But more impurity will go to solid at the same liquid concentration, because the segregation coefficient $K i$ becomes larger. Therefore, we expected the product obtained during batch 
crystallization to contain more impurity if the solid is harvested at a higher yield. However, after experiments $1,2,3,4$, and 5 the measured purity was substantially different, even though the yields of these operations were rather constant. This observation suggests that kinetic effect of impurities on crystal growth significantly determine the segregation and incorporation of the impurity in the main solid phase.

It is usually assumed that molecules adsorbed at the growing crystal interface need time to settle into their equilibrium positions, without which the formation of defects if the superficial crystal structure is likely to occur. Rapid growth raises the possibility of mis-orientations in the lattice, and therefore results in higher roughness of the crystal surface and increased impurity capture. This could explain the results of experiments 3,4 and 5: the crystals contained more impurity when grown at higher supersaturation. However, this is not true for experiments 1,2 and 3 .

In fact, the number of defects on the crystal surface also depends on the total area of the crystal, provided that the rate of crystal growth is not limited by film diffusion. The amount of impurity captured can therefore be expected to increase with increasing seed area. This could explain why among experiments 1,2 and 3, because of its largest seed area, experiment 1 yielded zinc lactate crystals with the highest impurity content. Moreover experiment 6 produced crystals containing more impurities than the other experiments. It should be noticed that experiment 6 was seeded with particles whose area was reduced by a factor of 2: this observation seems to contradict the previous assumption. However, it is clear that experiment 6 can hardly be compared to other experiments because fewer seeds were used and the final temperature was lower. As a result, high level of supersaturation was generated in the late period of operation. Such a situation favors secondary nucleation of a large amount of fine crystals, which is clearly evidenced by the sharp increase in the crystal number observed by the FBRM displayed in Figure 7. The relationship between the concentrations of impurity incorporated in the final particles and an SA given by Equation (3), tends to confirm the relationship between the seed surface area and the incorporation of impurity: the products of experiments 2-5 exhibit similar impurity concentration (i.e. $C_{i}$ is between $0.38 \%$ and $0.40 \%$ ) while experiment 1 , performed with a doubled seed area, is characterized by a higher impurity content (i.e. $C_{i}=0.44$ ). As outlined previously, experiment 7 was not taken into account here.

\section{III.3. Effect of the operating condition on the CSD}

Comparing the FBRM readings in Figure 7 shows that the crystal counts increase rapidly during experiments 1 and 3, while they remain almost constant during experiment 2. Actually, sharp increases in the count of crystals were only observed when small seed particles were used, i.e. in experiments 1, 3, 4 and 5. Increases in the crystal numbers could therefore result from "false" nuclei detached from the seed surface, or from seed crystals smaller than $0.5 \mu \mathrm{m}$ which could have been too small to be detected first, but counted after subsequent crystal growth. New particles could also result from secondary nucleation phenomena. Indeed, secondary nucleation is likely to occur according to various mechanisms, even at low levels of supersaturation; and it is reasonable to assume that secondary nucleation is enhanced by increasing seed surface area.

As mentioned above, large amount of new particles was produced during experiment 6 . High secondary nucleation rate can be explained by the high level of supersaturation obtained at time $t=8000 \mathrm{~s}$ : the peak observed in Figure 6 could be attributed to the fact that the limit curve of metastable zone was reached.

Now, in the absence of refined population balance equation model, the following rough estimation of an average particle size can be made

$N_{\text {seed }}=\frac{W_{\text {seed }}}{\phi_{\text {seed }} \rho_{s} \pi D_{\text {seed }}^{3}}$ 


$$
N_{\text {final }}=\frac{W_{\text {final }}}{\phi_{\text {seed }} \rho_{s} \pi D_{f \text {,exp }}^{3}}
$$

where $\rho_{s}$ is the density of zinc lactate, $D_{f, \exp }$ is an average size of the final crystallized particles, measured using laser diffraction technique, and $N_{\text {final }}$ is the final number of particles.

From the previous equations, assuming negligible secondary nucleation (i.e. $N_{\text {final }}=N_{\text {seed }}$ ) during the whole crystallization process leads to the following estimation of the final particle size

$$
D_{f, \text { cal }}=\left(\frac{W_{\text {final }}}{W_{\text {seed }}}\right)^{1 / 3} D_{\text {seed }}
$$

A rough estimation of the average particle size of final crystals $\left(D_{f, c a l}\right)$ can then be calculated on the basis of Equation (7). The results of such a computation are listed in Table 2.

Table 2 shows that the final particle sizes, $D_{f, \text { exp }}$, are smaller than the corresponding estimates $D_{f, c a l}$ computed assuming negligible nucleation during the cooling batch crystallization process. A quantitative measure of the ratio between the two particle sizes is computed as follows:

$$
\rho_{N}=\left(\frac{D_{f, \text { exp }}}{D_{f, \text { cal }}}\right)
$$

Table 2 shows that, except for experiment 6, the values of the ratio $\rho_{N}$ are rather comparable, which confirms that the previous guesses about the relationships between the seeding parameters and the final number of particle make some sense. Moreover $\rho_{N}>1$ was expected as the assumption made for Equation (7) clearly underestimates the final particle number (assuming that no secondary nucleation takes place). Moreover it is worth noting that according to the particle shapes and optical properties, one cannot consider the measured particle size distribution as fully reliable.

In similar vein, Figure 7 shows that the average level of supersaturation during experiment 3 was higher than that of experiment 1 . Considering the possible occurrence of secondary nucleation during the crystallization process, one would expect more secondary particles to be generated during experiment 3. This latter assumption is indeed confirmed in Figure 7, where the final FBRM counts of experiment 3 appear to be higher than half of those of experiment 1. To summarize the experimental results about the effect of operating conditions on the incorporation of impurities, it is clear that more experiments with various seeding parameters and cooling rates should be performed to provide a sufficient amount of data. However, Figure 8 summarizes the main trends that can be identified from the present work. First, it was checked that despite their roughness, estimates of the final particles size and particle numbers could be obtained from simple average mass balance equations. Second, it turns out that the final impurity concentration of the solid can be reduced with decreasing the seed particle number or increasing the final particle size. As these two parameters are clearly connected (i.e. increasing the number of seeds yields smaller final crystal), one can hardly draw clear conclusions from this latter observation, but it is likely that increasing the area of crystals during the batch crystallization process makes the incorporation of impurities easier.

III.4. Temperature-dependency of the yield of crystallization

As shown in Figure 6, the final relative supersaturation threshold depends on the final temperature. For example, the final zinc lactate concentrations at 35 and $45{ }^{\circ} \mathrm{C}$ were 2.83 and $3.28 \mathrm{~g} / 10 \mathrm{O} \mathrm{H}_{2} \mathrm{O}$, corresponding to relative supersaturation values of 0.1587 and 0.1264 , respectively.

According to Kubota [11], the final crystal growth rate can be inhibited according to the socalled "pinning mechanism". When compared to the growth of crystals in pure solvent, the time-averaged advancement velocity of a step in an impure media can be hindered by the 
adsorption of impurity species on the growing crystal surface. Indeed, during the step advancement kink sites can be blocked by foreign species that cannot easily be incorporated in the crystal lattice. To allow further crystal growth, the growth-step has to circumvent the adsorbed impurity, which obviously reduces the overall growth rate. Several models describing such "pinning mechanism" were early described in the literature [28]. It is worth noting that many convincing observations of the pinning mechanism were reported in the literature, notably thanks to the use of advanced imaging techniques such as Atomic Force Microscopy (AFM). Kubota-Mullin's model [29] was proposed to describe the pinning mechanism through the ratio $\Gamma$ between the step velocities in pure $\left(u_{0}\right)$ and impure $(u)$ solvents. $\Gamma$ is given by the following expression:

$$
\Gamma=\frac{u}{u_{0}}=1-\left[\frac{\gamma a}{K T \sigma L}\right] \theta=1-\alpha \theta^{*}
$$

where $\gamma$ is the edge free energy, $a$ is the size of the growth unit, $T$ is the absolute temperature, $k$ is the Boltzmann constant and $\theta^{*}$ is the steady-state coverage fraction of active growing crystal surface by adsorbed impurities.

In order to relate the step velocity to the growth rate, one generally assumes that $G \propto u$. The overall parameter $\alpha$ is the effectiveness factor quantifying the efficiency of the impurity specie in hindering the crystal growth. It is very important to notice that $\alpha$ depends not only on properties of the involved solid, but also on supersaturation. Several approaches can be used to compute the equilibrium coverage $\theta^{*}$. In Kubota-Mullin's model [29] and [30], the equilibrium coverage of the growing surface is estimated according to the Langmuir adsorption theory

$\theta^{*}=\frac{K C_{i}}{1+K C_{i}}$

where $\mathrm{K}$ is the Langmuir adsorption constant and $\mathrm{C}_{\mathrm{i}}$ is the concentration of impurity.

Equations (9) and (10) lead to the following expression of the final steady-state relative supersaturation

$$
\sigma_{c}=\frac{\gamma a K c}{K T L(1+K c)}
$$

Moreover according to Mersmann [31], $\gamma$ can be calculated as follows:

$$
\gamma=\kappa K T(C c N)^{2 / 3} \ln \left(\frac{C c}{C^{*}}\right)
$$

where $N$ is Avogadro number, and $C c$ the molar density of the crystallized solid in question. $\kappa$ is an empirical factor which was estimated between 0.310 and 0.414 from a large set of experimental data [32]. The surface tension is roughly proportional to the temperature, if one neglects small variation of $\ln \left(\mathrm{Cc} / \mathrm{C}^{*}\right)$ with temperature. Therefore, one has

$\gamma=\delta t$

Moreover the influence of temperature on the adsorption coefficient can be calculated on the basis of Gibbs equation

$$
\ln K=-\frac{\Delta H^{0}}{R T}+\frac{\Delta S^{0}}{R}
$$

Combining Equations (11), (13) and (14) yields 


$$
\frac{1}{\sigma c}=A\left[1+\frac{1}{c} e^{-(m / T+n)}\right]
$$

where $\mathrm{A}=\mathrm{kL} / \delta \mathrm{a}, \mathrm{m}=-\Delta \mathrm{H}^{\circ} / \mathrm{R}$ and $\mathrm{n}=\Delta \mathrm{S}^{\circ} / \mathrm{R}$.

In order to assess the relevance of Equation (15), the reciprocal of the final measured supersaturation is plotted as a function of the reciprocal of temperature in Figure 9. The experimental data turn out to fit quite well Equation (11) and allow estimating the following parameters values

$$
\frac{1}{\sigma c}=4.74+96.15 e^{-(7020.1 / T-18.18)}
$$

Thus, the standard adsorption heat of malic acid on the surface of zinc lactate crystals is determined to be $-58.37 \mathrm{~kJ} / \mathrm{mol}$; such adsorption may be caused by the formation of metal-organic complexes [32].

\section{III.5. Crystal habit and structure}

The final products of experiments 1 and 6, which correspond to the highest impurity concentrations, were investigated by SEM and XRD so as to analyse possible modifications of shape and structure with respect to pure zinc lactate crystals. As displayed in Figure 10, the pure zinc lactate crystals exhibit a cuboid shape, while impure crystals are characterized by more elongated and thinner shapes. This indicates that malic acid molecules were selectively adsorbed on specific faces of zinc lactate crystals. It is recalled here that the size of crystals from experiment 6 was larger than the size of crystals obtained from experiment 1.

Figure 11 compares the XRD spectra of pure and impure zinc lactate crystals. The positions of the main peaks, which reflect the symmetry and size of unit cell, are almost the same, indicating negligible changes of the cell parameter by possible incorporation of impurity molecules. However, some relative peak intensities appear to be rather altered, which is an indication of differences in the orientation of the unit cell's alignment.

\section{Conclusions}

In this study, the solution crystallization of zinc lactate in the presence of malic acid was investigated. Malic acid acted as an impurity during the crystallization process, and was found to increase significantly the solubility of zinc lactate and to delay the onset of primary nucleation. The effect of seed amount and size was studied. It appears that an appropriate selection of the seeding parameters allows obtaining final products with higher average crystal size and better chemical purity, but a more extensive approach of this important industrial problem would clearly require advanced dynamic crystallization models to be developed. Anyway, the final purity of crystals was found to depend on the particle sizes, (i.e. both seed sizes and final crystal sizes) probably because increased crystal area in suspension favours the adsorption of impurities during the growth of crystals. Other operating parameters such as seeding temperature, cooling rate and final temperature were also studied and shown to exert some influence on the crystallization process and on the effects of impurities.

Besides, as predicted by Kubota-Mullin's inhibition model, residual supersaturation thresholds were observed when the crystallization was performed in the presence of impurities. The effect of the final temperature on the supersaturation threshold was shown to obey a simple exponential expression derived from Kubota-Mullin's and Gibbs's equations. Some differences were observed between the structure of crystals grown in impure media and the structure of pure crystals, even though potential amounts of malic acid incorporated in the crystal structure could not be quantified through XRD. 


\section{Acknowledgements}

This work has been supported by NSFC (20476026), 111 Project (Bo8021) and Open project of Skloche (SKL-ChE-o8Co8).

The French research agency ANR is also acknowledged for the support granted to the project "IPAPI" (Improving the Properties of Active Pharmaceutical Ingredients), Ref. o7-BLAN0183.

\section{References}

[1] M. Fujiwara, Z.K. Nagy, J.W. Chew and R.D. Braatz, Chemical Engineering Science 59 (2004), pp. 313-327.

[2] N. Blagden, M. de Matas, P.T. Gavan and P. York, Advanced Drug Delivery Reviews 59 (2007), pp. 617-630.

[3] N. Doki, N. Kubota and A. Sato, Chemical Engineering Journal 81 (2001), pp. 313-316.

[4] P.A. Meenan, S.R. Andrson and D.L. Klug, The influence of impurities and solvents on crystallization. In: A.S. Myerson, Editor, Handbook of Industrial Crystallization (second ed.), Elsevier Science \& Technology Books, Amsterdam (2002) pp. 67-100.

[5] A.A. Chernov, Modern Crystallography III. Crystal Growth, Springer-Verlag, Berlin (1984).

[6] E. Kirkova, M. Djarova and B. Donkova, Progress in Crystal Growth and Characteristics 32 (1996), p. 111.

[7] N. Kubota, I. Uchiyama, K. Nakai, K. Shimizu and J.W. Mullin, Industrial and Engineering Chemistry Research 27 (1988), pp. 930-934.

[8] G.G.Z. Zhang and D.J.W. Grant, International Journal of Pharmaceutics 181 (1999), pp. 61-70.

[9] R. Meadhra and R. Lin, Chemical Engineering Research and Design 84 (2006), pp. 711-720.

[10] F. Rosenberger and H.G. Riveros, Journal of Chemical Physics 60 (1974), pp. 668-670.

[11] N. Kubota, Crystal Research and Technology 36 (2001), p. 749.

[12] K. Sangwal and E. Mielniczek-Brzóska, Fluid Phase Equilibria 258 (2007), pp. 199-205.

[13] W.M.L. Wood, Powder Technology 121 (2001), pp. 53-59.

[14] K. Sangwal and E. Mielniczek-Brzóska, Journal of Crystal Growth 267 (2004), pp. 662675.

[15] M. Bravi and B. Mazzarotta, Chemical Engineering Journal 70 (1998), pp. 197-202.

[16] L.N. Rashkovich and N.V. Kronsky, Journal of Crystal Growth 182 (1997), pp. 434-441.

[17] J. Dugua and B. Simon, Journal of Crystal Growth 44 (1978), pp. 280-286.

[18] I. Weissbuch, M. Lahav and L. Leiserowitz, Advances in Crystal Growth Research 43 (2001), pp. 381-400.

[19] J.C. Givand, A.S. Teja and R. Rousseau, Journal of Crystal Growth 198/199 (1999), pp. 1340-1344.

[20] Z.Q. Yu, P.S. Chow and B.H.T. Reginald, Industrial and Engineering Chemistry Research 45 (2006), pp. 438-444.

[21] G. Cao and M.J. Shah, Microporous and Mesoporous Materials 101 (2007), pp. 19-23.

[22] A.I. Vogel, Textbook of Quantitative Inorganic Analysis (third ed.), Longman, London (1961).

[23] E.L. Shock and C.M. Koretsky, Geochimica et Cosmochimica Acta 57 (1993), pp. 48994922.

[24] T.H. Giordano, The Role of Organic Acids in Geological Processes, Springer-Verlag (1994) pp. 319-354.

[25] R.N. Hall, Journal of Physical Chemistry 57 (1953), p. 836.

[26] K.J. Weiser, Physics and Chemistry of Solids 7 (1958), pp. 118-126.

[27] J.A. Burton, R.C. Prim and W.P. Slichter, Journal of Chemical Physics 21 (1953), pp. $1987-1996$.

[28] N. Cabrera, D.A. Vermilyea In Growth and Perfection of Crystals, New York, 1958, pp. 393. 
Journal of Crystal Growth, 2010, 312(19), 2747-2755, doi:10.1016/j.jcrysgro.2010.06.005

[29] N. Kubota and J.W. Mullin, Journal of Crystal Growth 152 (1995), pp. 203-208.

[30] N. Kubota, M. Yokota and J.W. Mullin, Journal of Crystal Growth 182 (1997), pp. 8694.

[31] A. Mersmann, Crystallization Technology Handbook (second ed.), Marcel Dekker, New York (2001).

[32] B.O. Von, W. Kordel and W. Klein, Chemosphere 22 (1991), pp. 285-304.

\section{Figures}

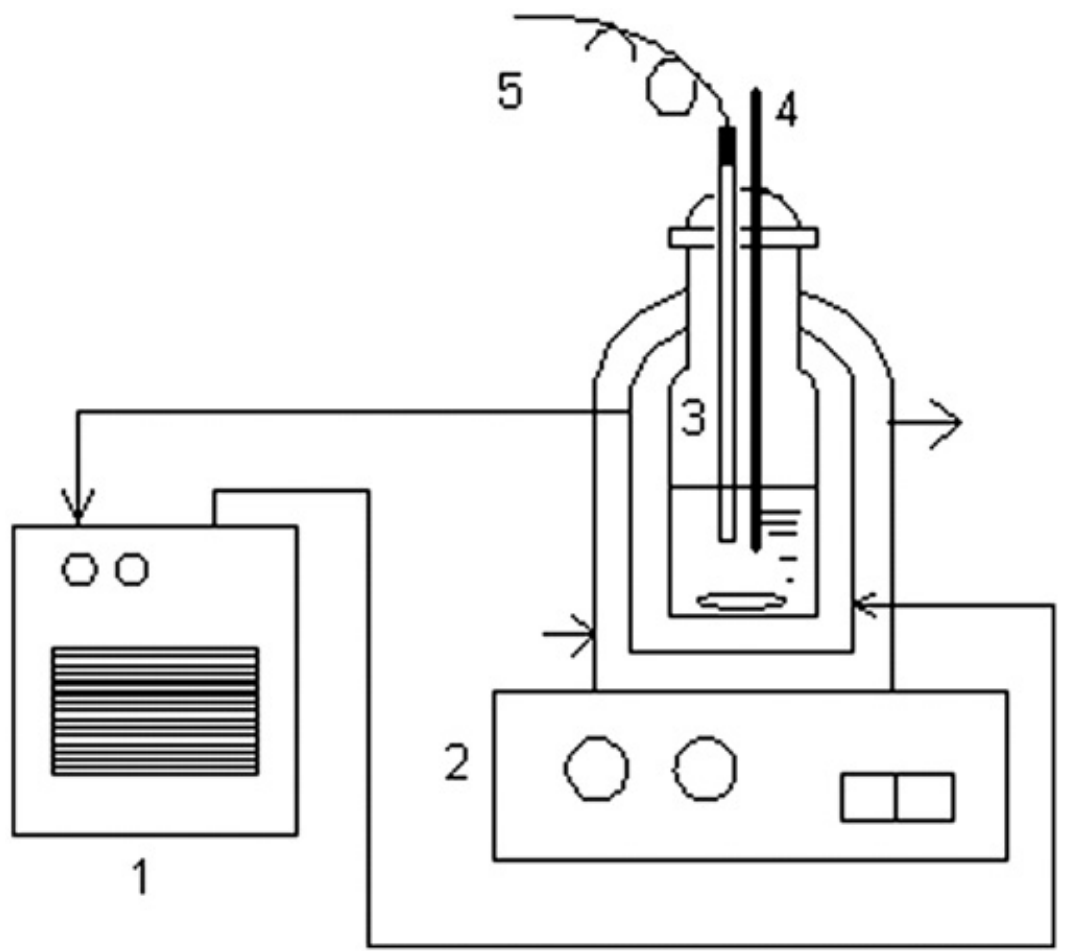

Figure 1: Schematic diagram of experimental apparatus for measurements of solubility. (1) Thermostat; (2) magnetic stirrer; (3) double-jacketed vessel; (4) thermometer; (5) pH meter.

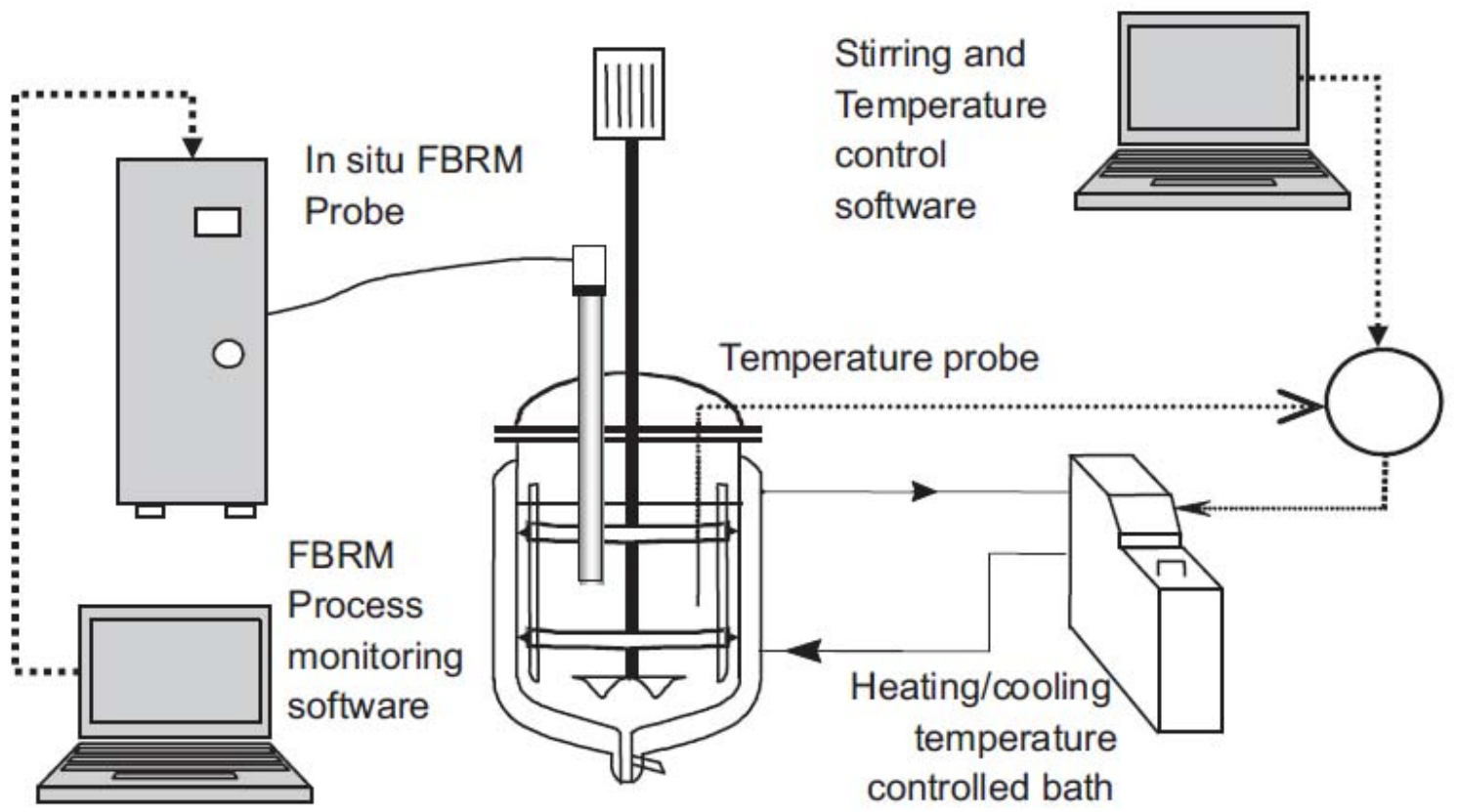

Figure 2: Experimental setup for batch cooling seeded experiments. 

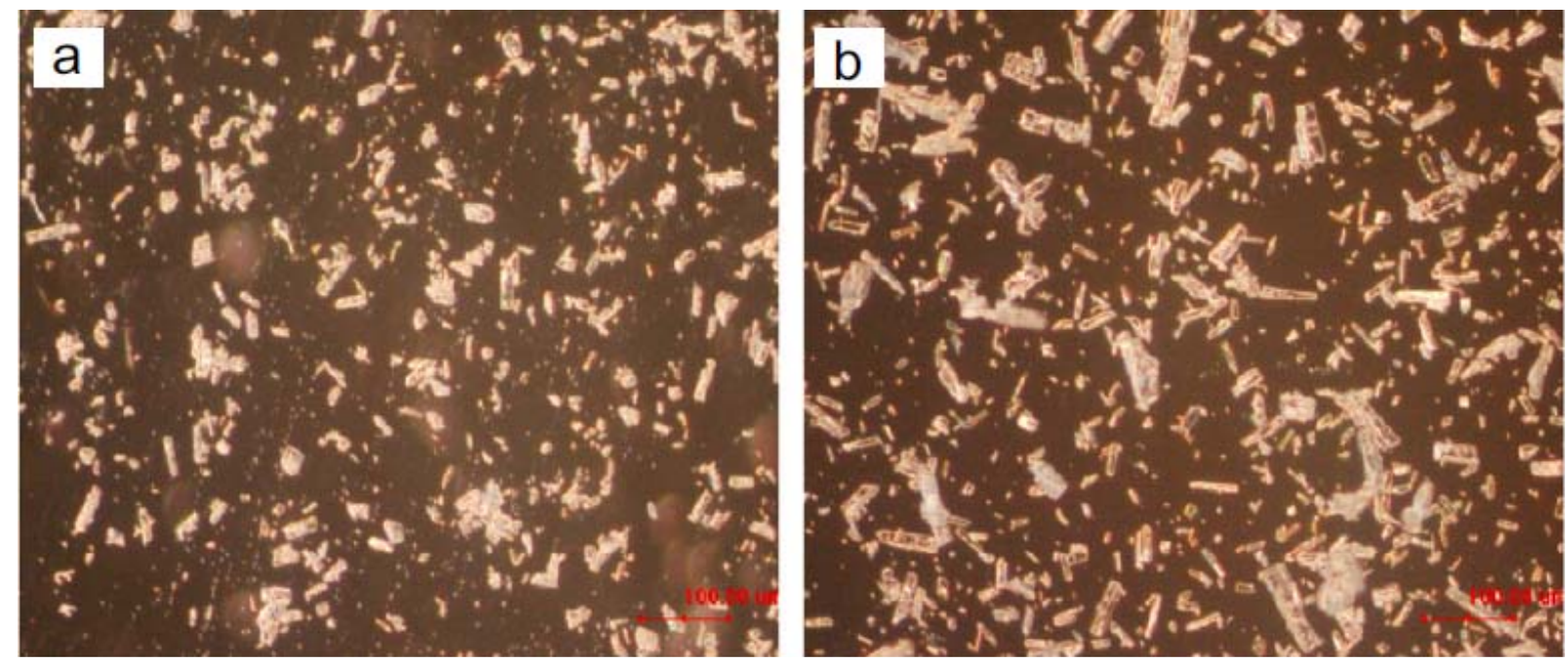

Figure 3: Microscope images of different seeds generated by grinding: (a) small seeds; (b) big seeds.

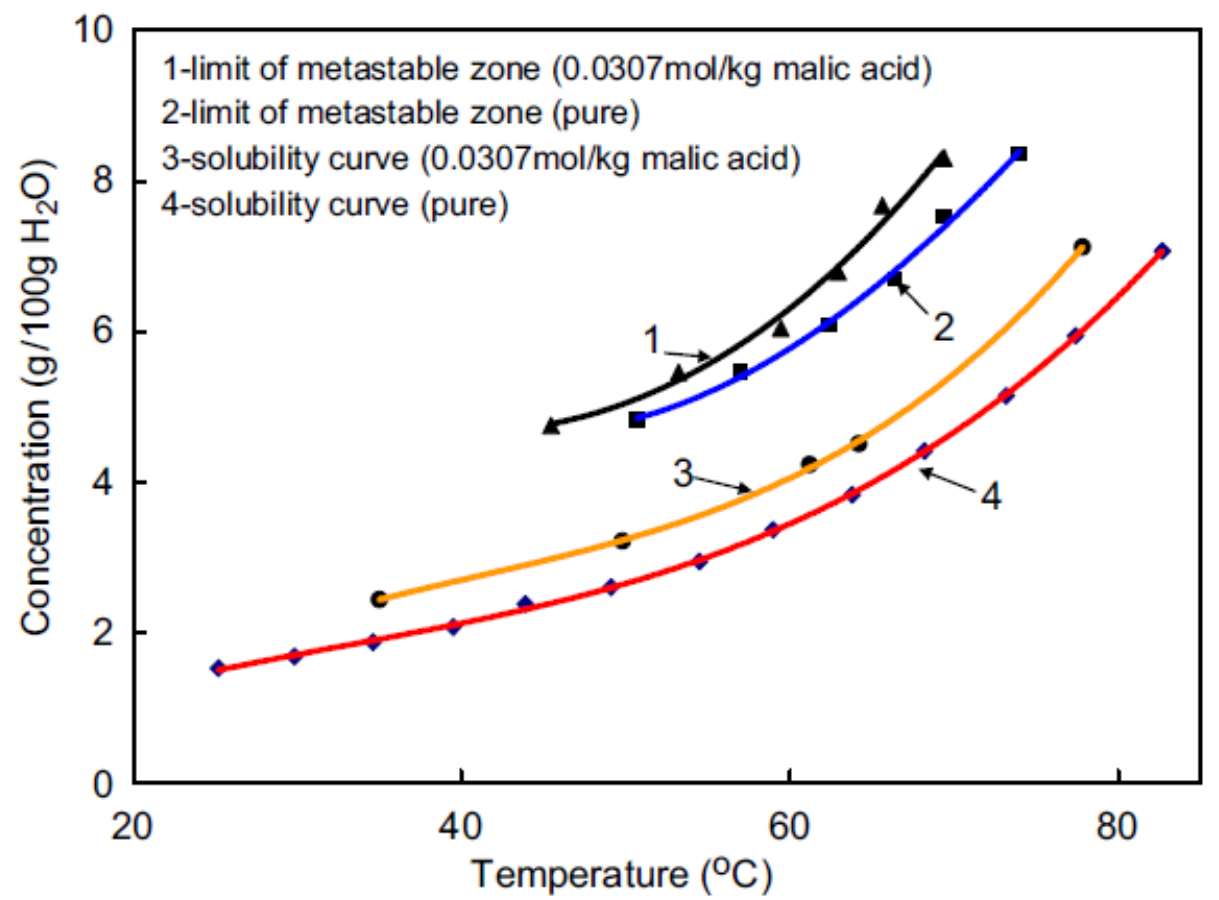

Figure 4: Effect of the impurity on the solubility and limit of metastable zone of zinc lactate (20o rpm, cooling rate $=-10^{\circ} \mathrm{C} / \mathrm{h}$ ). 


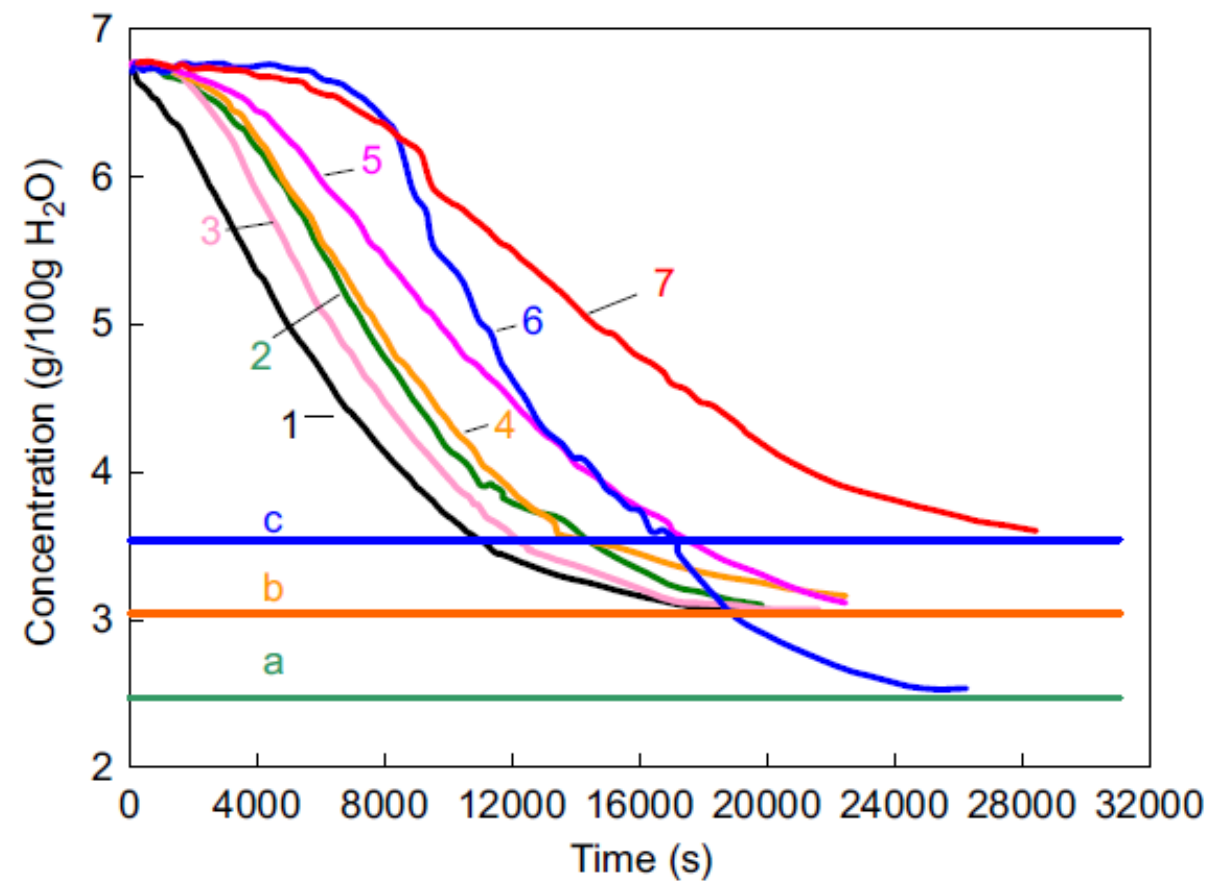

Figure 5: Time variations of zinc lactate concentration under different crystallization conditions ( $a, b$, and $c$ indicating the final concentration at $3 \mathrm{O}, 4 \mathrm{O}$ and $5 \mathrm{O}^{\circ} \mathrm{C}$ ).

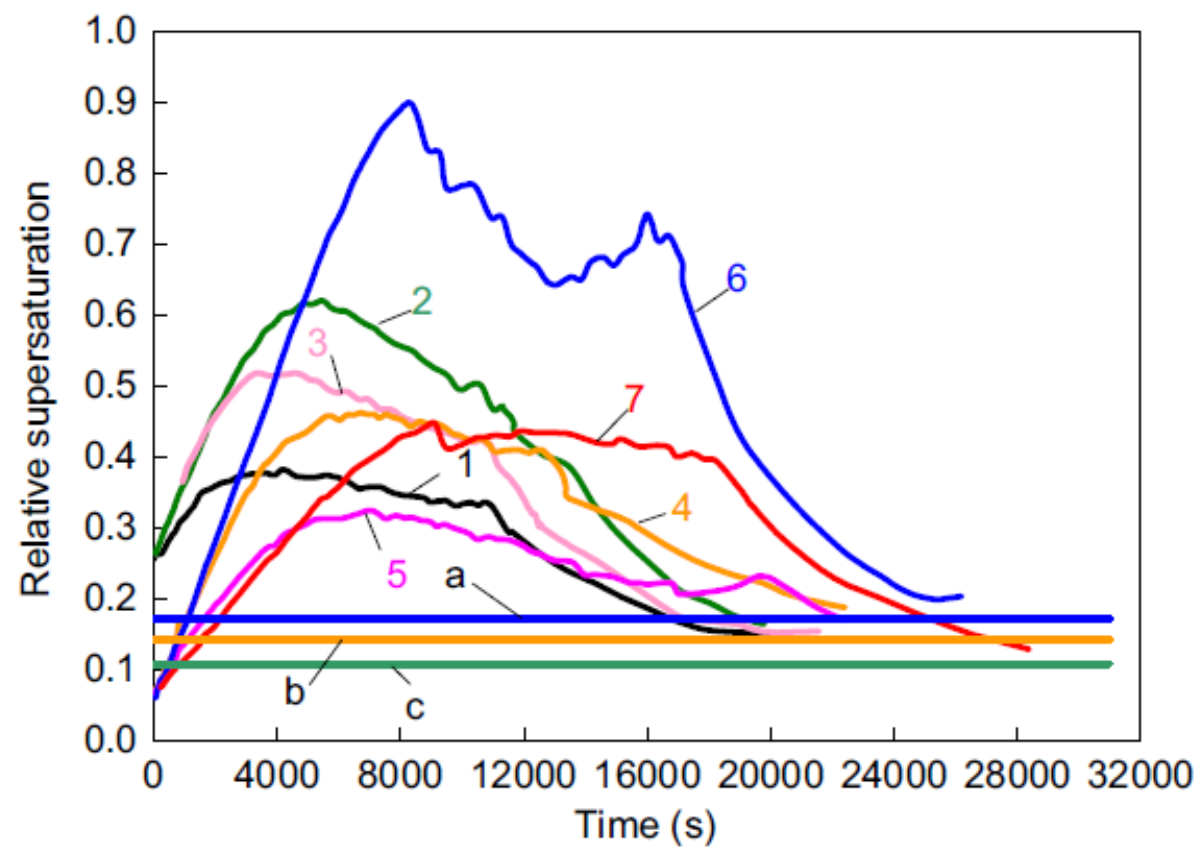

Figure 6: Time variations of the relative supersaturation of zinc lactate during cooling crystallization ( $a, b$, and $c$ indicating the final supersaturations at 30,40 and $50^{\circ} \mathrm{C}$ ). 


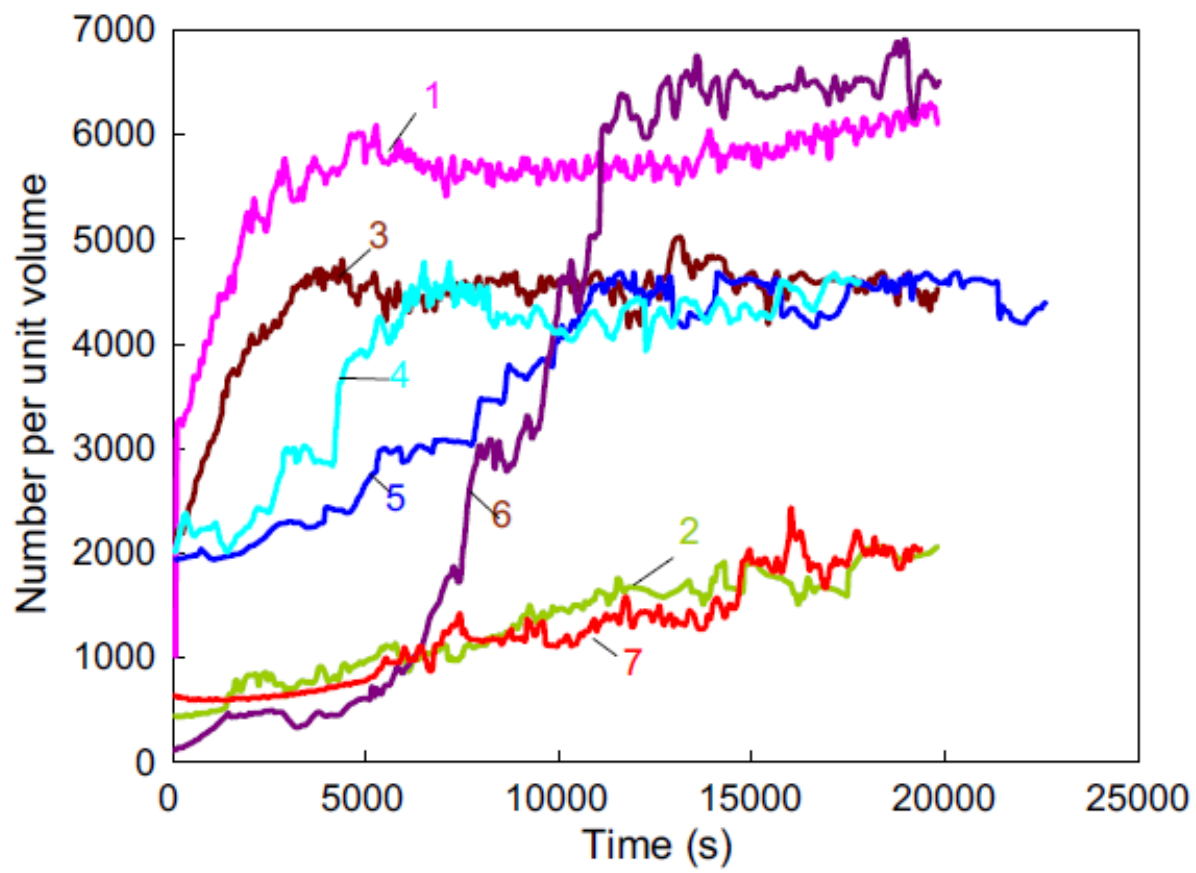

Figure 7: Variation of an FBRM reading during the crystallization experiments.

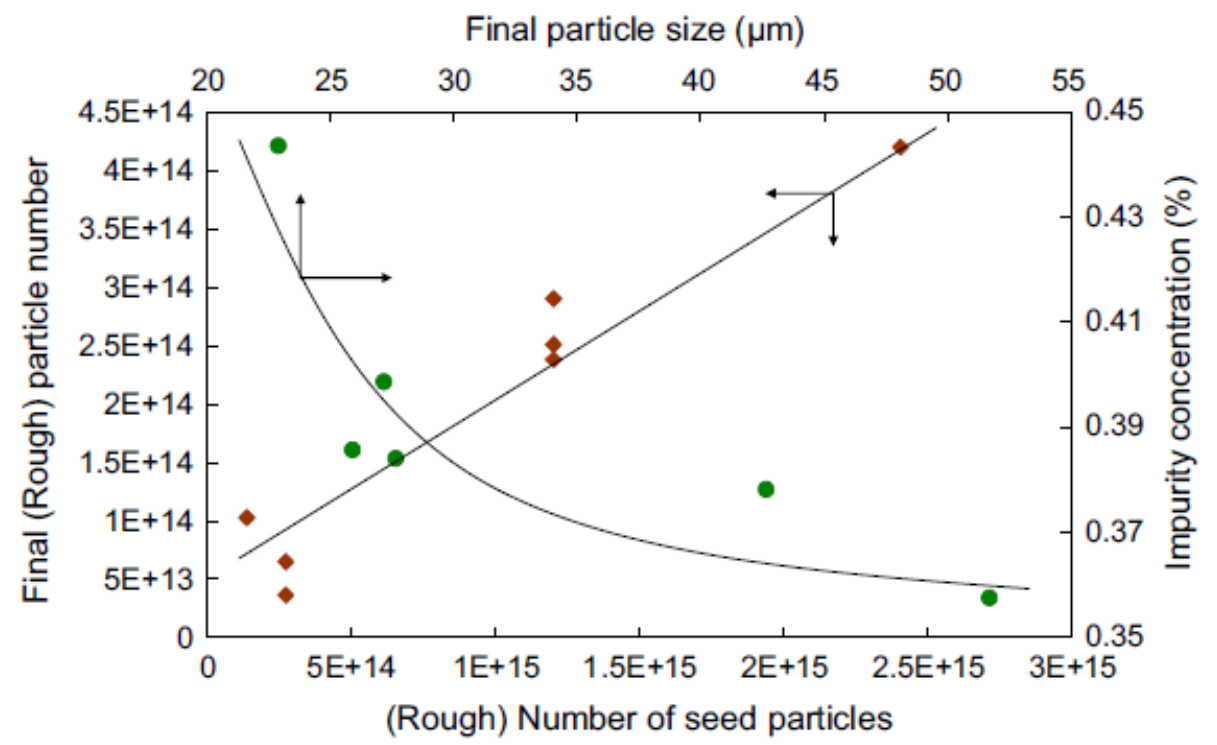

Figure 8: Experimental relationship between the estimated number of seed particles (Equation (5)) and the final number of zinc lactate crystals (lozenges), and effect of the final particle size on the final impurity concentration (circles). 
Journal of Crystal Growth, 2010, 312(19), 2747-2755, doi:10.1016/j.jcrysgro.2010.06.005

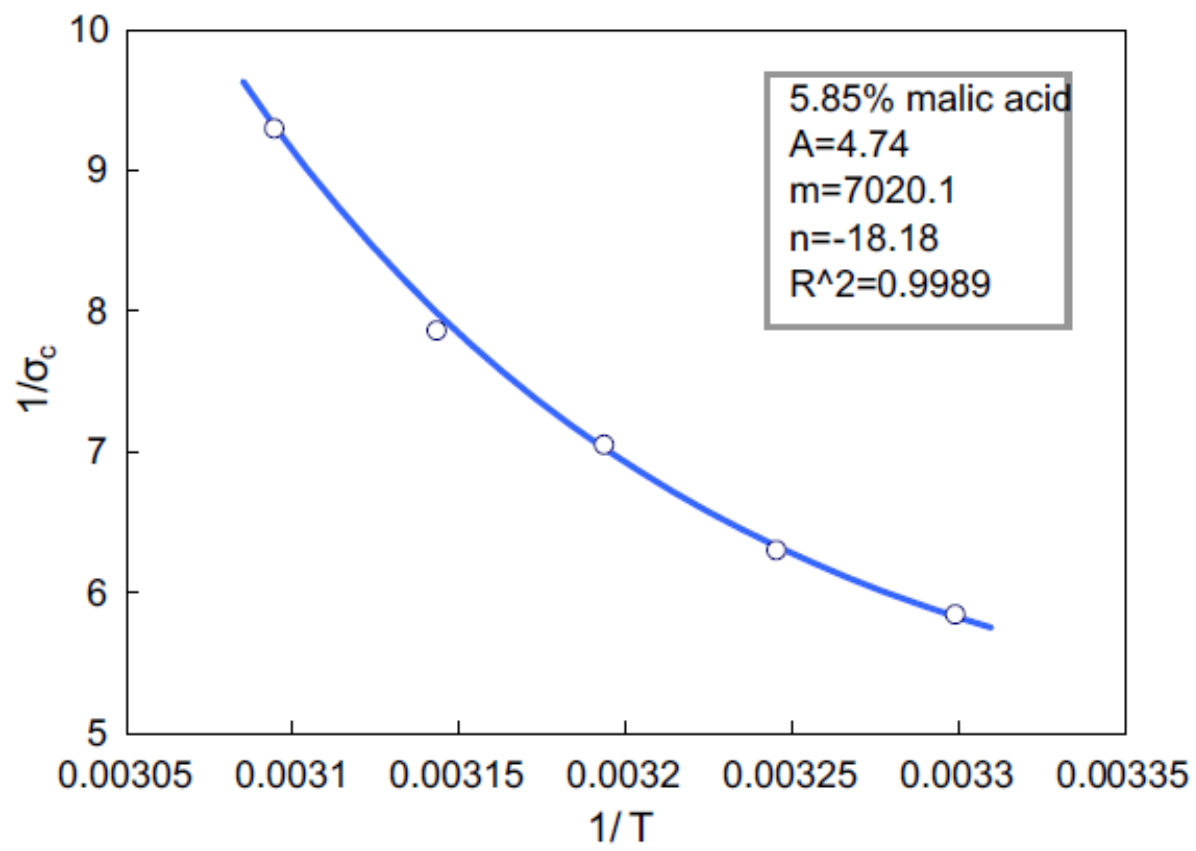

Figure 9: The reciprocal of the final supersaturation threshold as a function of the reciprocal of temperature in presence of 5.85 wt\% malic acid. Dots: experiments; line: fitting by Equation (15).
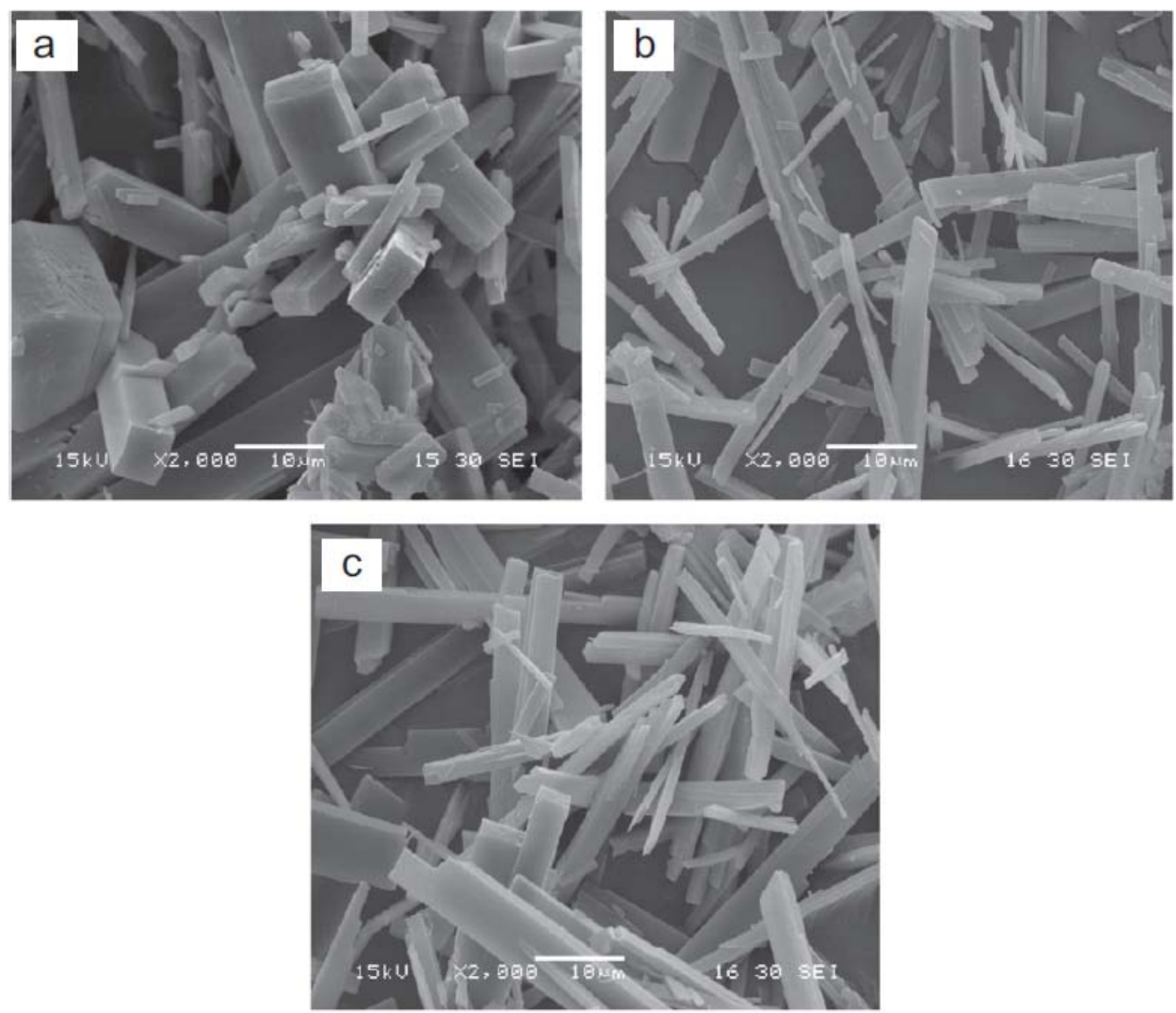

Figure 10: SEM images of zinc lactate crystals: (a) pure zinc lactate; (b) impure zinc lactate from Exp. 1; (c) impure zinc latate from Exp. 6. 
Journal of Crystal Growth, 2010, 312(19), 2747-2755, doi:10.1016/j.jcrysgro.2010.06.005

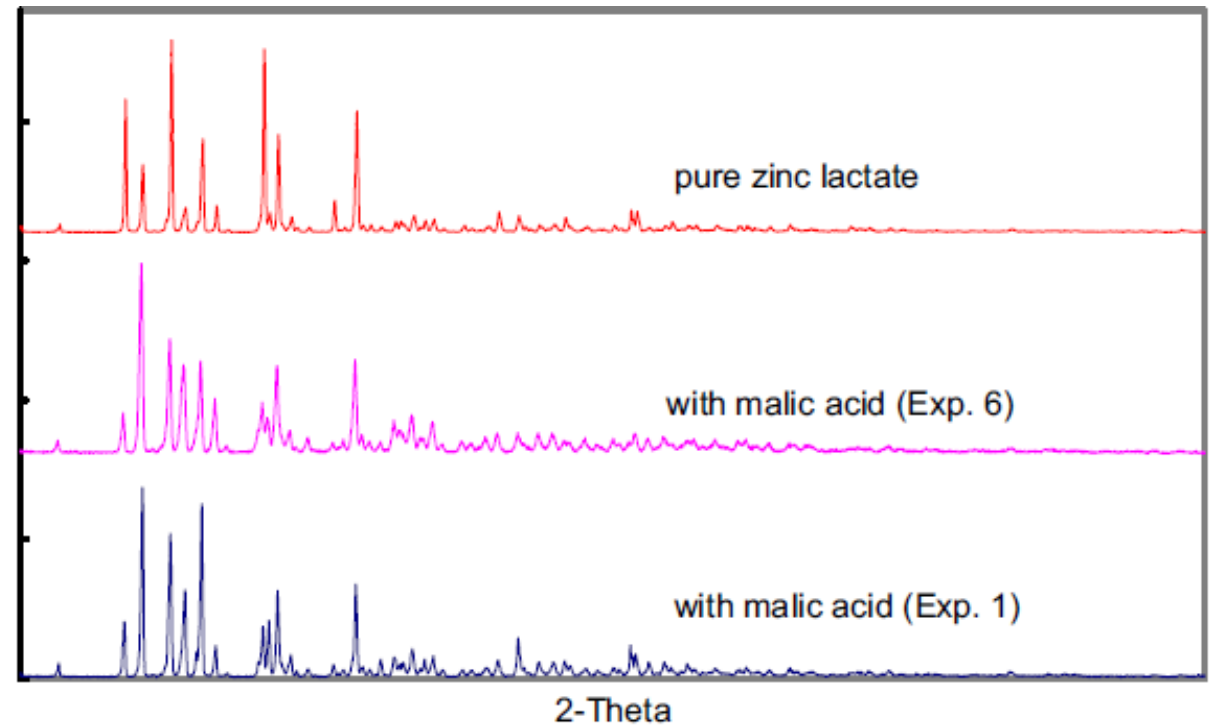

Figure 11: Comparison of XRD patterns of pure and impure zinc lactate crystals. 
Journal of Crystal Growth, 2010, 312(19), 2747-2755, doi:10.1016/j.jcrysgro.2010.06.005

\section{Tables}

Table 1: Crystallization of zinc lactate in the presence of malic acid: main experimental results.

\begin{tabular}{|c|c|c|c|c|c|c|c|c|c|}
\hline $\mathrm{N}^{\circ}$ & $\begin{array}{c}W_{\text {seed }} \\
(\%)\end{array}$ & $\begin{array}{c}D_{\text {seed }} \\
(\mu \mathrm{m})\end{array}$ & $T_{s}\left({ }^{\circ} \mathrm{C}\right)$ & $\begin{array}{c}D_{f, \exp } \\
(\mu \mathrm{m})\end{array}$ & Yield $(\%)$ & $\begin{array}{c}C_{\text {final }} \\
(\mathrm{g} / 10 \mathrm{~g} \\
\left.\mathrm{H}_{2} \mathrm{O}\right)\end{array}$ & $C_{i}(\%)$ & $\begin{array}{c}\text { Cooling } \\
\text { rate } \\
\left({ }^{\circ} \mathrm{C} / \mathrm{h}\right)\end{array}$ & $\begin{array}{c}T_{\text {final }} \\
\left({ }^{\circ} \mathrm{C}\right)\end{array}$ \\
\hline 1 & 2 & 9.4 & 70 & 22.89 & 54.69 & 3.04 & 0.4436 & -10 & 40 \\
\hline 2 & 2 & 19.4 & 70 & 42.66 & 55.03 & 3.04 & 0.3782 & -10 & 40 \\
\hline 3 & 1 & 9.4 & 70 & 27.17 & 55.03 & 3.04 & 0.3987 & -10 & 40 \\
\hline 4 & 1 & 9.4 & 75 & 25.89 & 55.1 & 3.04 & 0.3857 & -10 & 40 \\
\hline 5 & 1 & 9.4 & 75 & 27.65 & 55.1 & 3.04 & 0.3841 & -7.7 & 40 \\
\hline 6 & 1 & 19.4 & 75 & 36.62 & 63.52 & 2.47 & 0.6131 & -10 & 30 \\
\hline 7 & 2 & 19.4 & 75 & 51.71 & 47.71 & 3.54 & 0.3575 & -5 & 50 \\
\hline
\end{tabular}

Table 2: Rough estimates of seed and final particle features.

\begin{tabular}{|c|c|c|c|c|c|}
\hline $\mathrm{N}^{\circ}$ & $\begin{array}{c}W_{\text {seed }} \\
(\%)\end{array}$ & $\begin{array}{c}D_{\text {seed }} \\
(\mu \mathrm{m})\end{array}$ & $\begin{array}{c}\text { SA (arbitrary } \\
\text { units })\end{array}$ & $\begin{array}{c}D_{f, c a l} \\
(\mu \mathrm{m})\end{array}$ & $\begin{array}{c}\mathrm{D}_{\text {f,exp }} \\
(\mu \mathrm{m})\end{array}$ \\
\hline 1 & 2.0 & 9.4 & 0.213 & 28.32 & 22.89 \\
\hline 2 & 2.0 & 19.4 & 0.103 & 58.57 & 42.66 \\
\hline 3 & 1.0 & 9.4 & 0.106 & 35.75 & 27.17 \\
\hline 4 & 1.0 & 9.4 & 0.106 & 35.77 & 25.89 \\
\hline 5 & 1.0 & 9.4 & 0.106 & 35.77 & 27.65 \\
\hline 6 & 1.0 & 19.4 & 0.052 & 77.41 & 36.62 \\
\hline 7 & 2.0 & 19.4 & 0.103 & 55.85 & 51.71 \\
\hline
\end{tabular}

